PROCEEDINGS OF THE

AMERICAN MATHEMATICAL SOCIETY

Volume 135, Number 4, April 2007, Pages 1129-1132

S 0002-9939(06)08563-7

Article electronically published on October 11, 2006

\title{
INVERSE SPECTRAL THEORY FOR STURM-LIOUVILLE PROBLEMS WITH FINITE SPECTRUM
}

\author{
HANS VOLKMER AND ANTON ZETTL \\ (Communicated by Carmen C. Chicone) \\ Dedicated to the memory of F.V. Atkinson
}

\begin{abstract}
For any positive integer $n$ and any given $n$ distinct real numbers we construct a Sturm-Liouville problem whose spectrum is precisely the given set of $n$ numbers. Such problems are of Atkinson type in the sense that the weight function or the reciprocal of the leading coefficient is identically zero on at least one subinterval.
\end{abstract}

\section{INTRODUCTION}

In this paper we show that, for any $n \in \mathbb{N}=\{1,2,3, \ldots\}$ and any given real numbers

$$
\lambda_{1}<\lambda_{2}<\cdots<\lambda_{n}
$$

there exists a regular self-adjoint Sturm-Liouville problem consisting of an equation

$$
-\left(p y^{\prime}\right)^{\prime}+q y=\lambda w y \text { on } J=(a, b),-\infty<a<b<\infty,
$$

with coefficients satisfying

$$
\frac{1}{p}, q, w \in L^{1}(J, \mathbb{R})
$$

and separated boundary conditions

$$
\begin{gathered}
\cos \alpha y(a)-\sin \alpha\left(p y^{\prime}\right)(a)=0,0 \leq \alpha<\pi, \\
\cos \beta y(b)-\sin \beta\left(p y^{\prime}\right)(b)=0,0<\beta \leq \pi,
\end{gathered}
$$

whose spectrum $\sigma$ is given by

$$
\sigma=\left\{\lambda_{j}: j=1,2, \ldots, n\right\} .
$$

If, in addition to (1.3), the coefficients satisfy

$$
p>0, w>0 \text { a.e. on } J,
$$

then this is a classical Sturm-Liouville problem which is well known to have a spectrum consisting of an infinite but countable number of real eigenvalues $\left\{\lambda_{n}\right.$ : $n \in \mathbb{N}=\{1,2,3, \ldots\}\}$ which can be ordered to satisfy

$$
-\infty<\lambda_{1}<\lambda_{2}<\lambda_{3}<\ldots
$$

Received by the editors March 21, 2005 and, in revised form, November 11, 2005. 2000 Mathematics Subject Classification. Primary 34B24, 34B09; Secondary 34L05.

Key words and phrases. Sturm-Liouville problems, finite spectrum, inverse spectral theory.

(C)2006 American Mathematical Society 
The classical theory of Sturm-Liouville problems was extended by Atkinson in 1]. Let

$$
r=\frac{1}{p} .
$$

Atkinson observed that the basic theory for equation (1.2) holds if the conditions (1.6) are relaxed to

$$
r \geq 0, w \geq 0 \text { on } J
$$

thus allowing $r=1 / p$ and $w$ to be identically zero on one or more subintervals of $J$. Theorem 8.4.6, p. 215 in [1] contains the following result.

Proposition 1. Let (1.3), (1.9) hold. Assume that for some $c, d \in J$,

$$
\int_{a}^{c} w>0, \int_{d}^{b} w>0, \int_{a}^{b} r>0 .
$$

If $r$ and $w$ are positive on a common subinterval of $J$, then the Sturm-Liouville problem (1.2), (1.4) has an infinite number of eigenvalues satisfying (1.7).

The statement on page 212 of [1], 'The eigenvalues ... form a sequence $\lambda_{0}<$ $\lambda_{1}<\ldots$, possibly finite ...', strongly suggests that Atkinson realized that without the hypotheses of Proposition 1, there may be only a finite number of eigenvalues, but he gave no proof or example. This was confirmed by Kong, Wu and Zettl [4] who showed that for any positive integer $n$ there exist such problems with exactly $n$ eigenvalues. It is also shown in 4 that, given any $n$ disjoint open intervals of the real line there exists a problem of Atkinson type which has exactly one eigenvalue in each of the given $n$ open intervals. But the construction in [4 does not directly yield a problem with pre-assigned eigenvalues.

Definition 1. If $r$ or $w$ is identically zero on one or more subintervals of $J$, we say that the equation (1.2), or the boundary value problem (1.2), (1.4) is of 'Atkinson type'. It is convenient to think of such problems in terms of their system formulation:

$$
u^{\prime}=r v, v^{\prime}=(q-\lambda w) u ; u=y, v=p u^{\prime} .
$$

Remark 1. When $r$ is identically zero on a subinterval then, of course, this means that $p$ is infinite on this subinterval. Notation can be a psychological barrier. We suspect that the universally used notation for equation (1.1) has been an obstacle preventing Atkinson's ideas and results from being more widely used. Atkinson suggests the notation

$$
-\left(\frac{1}{p} y^{\prime}\right)^{\prime}+q y=\lambda w y .
$$

Although this notation is more suggestive since the solutions depend on $1 / p$, not $p$, it has not been widely adopted.

\section{An inVERSE SPECTRAL CONSTRUCTION}

Our main result is

Theorem 1. Let $n \in \mathbb{N}$. Given any $n$ real numbers $\lambda_{1}<\lambda_{2}<\cdots<\lambda_{n}$ there exists a Sturm-Liouville problem of Atkinson type and with Dirichlet boundary conditions

$$
y(a)=0=y(b)
$$

whose spectrum is precisely the set $\left\{\lambda_{j}: j=1,2, \ldots, n\right\}$. 
Proof. First we observe that we may assume that $\lambda_{1}>0$ since the eigenvalues can be shifted by adding kwy to both sides of equation (1.2). The proof consists of two parts: (i) we construct two matrix eigenvalue problems, one of which has $\left\{\lambda_{j}: j=1,2, \ldots, n\right\}$ as its eigenvalues, and (ii) using a method of Atkinson we construct a Sturm-Liouville problem which is equivalent to this matrix eigenvalue problem.

Choose numbers $\left\{\lambda_{j}^{\prime}: j=1,2, \ldots, n\right\}$ such that

$$
0<\lambda_{1}^{\prime}<\lambda_{1}<\lambda_{2}^{\prime}<\lambda_{2}<\cdots<\lambda_{n}^{\prime}<\lambda_{n} .
$$

By Gantmacher and Krein [3, Supplement II], there exist unique numbers

$$
m_{1}, m_{2}, \ldots, m_{n}>0
$$

and

$$
l_{0}, l_{1}, l_{2}, \ldots, l_{n}>0, \sum_{j=0}^{n} l_{j}=1
$$

such that $\left\{\lambda_{j}: j=1,2, \ldots, n\right\}$ are the eigenvalues of the matrix eigenvalue problem

$$
A z=\lambda M z,
$$

and $\left\{\lambda_{j}^{\prime}: j=1,2, \ldots, n\right\}$ are the eigenvalues of the matrix eigenvalue problem

$$
A^{\prime} z=\lambda M z
$$

where $M$ is the diagonal matrix

$$
M=\operatorname{diag}\left(m_{1}, m_{2}, \ldots, m_{n}\right)
$$

$A$ is the tridiagonal matrix

$$
A=\left[\begin{array}{ccccccccc}
l_{0}^{-1}+l_{1}^{-1} & -l_{1}^{-1} & 0 & 0 & \cdots & 0 & 0 & 0 & 0 \\
-l_{1}^{-1} & l_{1}^{-1}+l_{2}^{-1} & -l_{2}^{-1} & 0 & \cdots & 0 & 0 & 0 & 0 \\
\cdots & \cdots & \cdots & \cdots & \cdots & \cdots & \cdots & \cdots & \cdots \\
0 & 0 & 0 & 0 & \cdots & 0 & -l_{n-2}^{-1} & l_{n-2}^{-1}+l_{n-1}^{-1} & -l_{n-1}^{-1} \\
0 & 0 & 0 & 0 & \cdots & 0 & 0 & -l_{n-1}^{-1} & l_{n-1}^{-1}+l_{n}^{-1}
\end{array}\right]
$$

and $A^{\prime}$ agrees with $A$ except for the entry in the last row and last column which is replaced by $l_{n-1}^{-1}$.

We now construct a Sturm-Liouville problem whose spectrum is the set of eigenvalues of the matrix problem (2.3). Let

$$
b=1+\sum_{j=1}^{n} m_{j}
$$

and consider the partition

$$
0=b_{0}<a_{1}<b_{1}<a_{2}<b_{2}<\cdots<a_{n}<b_{n}<a_{n+1}=b,
$$

where

$$
a_{1}=l_{0}, b_{k}=a_{k}+m_{k}, a_{k+1}=b_{k}+l_{k} \quad \text { for } k=1,2, \ldots, n .
$$

Let $K$ be the union of the intervals $\left[a_{k}, b_{k}\right]$ for $k=1,2, \ldots, n$. For example if $n=2$,

$$
K=\left[l_{0}, l_{0}+m_{1}\right] \cup\left[l_{0}+m_{1}+l_{1}, l_{0}+m_{1}+l_{1}+m_{2}\right] .
$$

The lengths of these two intervals are $m_{1}$ and $m_{2}$ and the complementary intervals of $K$ have lengths $l_{0}, l_{1}, l_{2}$. 
Let $\varkappa_{K}$ denote the characteristic function of $K$, i.e.,

$$
\varkappa_{K}(t)=\left\{\begin{array}{l}
1, \quad t \in K \\
0, \quad t \in[0, b] \backslash K .
\end{array}\right.
$$

Now we consider the following problem of Atkinson type:

$$
u^{\prime}=\left(1-\varkappa_{K}\right) v, v^{\prime}=-\lambda \varkappa_{K} u, u(0)=0=u(b) .
$$

Such problems have been studied by Volkmer in [5], 6].

We claim that the spectrum of (2.5) is precisely the set $\left\{\lambda_{j}: j=1,2, \ldots, n\right\}$. To prove the claim we write (2.5) as a difference equation following the same method Atkinson used on pp. 203, 204 of [1]. Let $u, v$ be a solution of (2.5). We note that $u$ and $v$ are alternately constant on the intervals of the partition (2.4), say $u(x)=u_{k}$ on $\left[a_{k}, b_{k}\right]$ and $v(x)=v_{k}$ on $\left[b_{k}, a_{k+1}\right]$. We add the definitions $u_{0}=u(a)=0$ and $u_{n+1}=u(b)=0$. By integrating the equations in (2.5) over suitable subintervals of the partition we obtain

$$
\begin{aligned}
v_{k}-v_{k-1}=-\lambda u_{k} m_{k} & \text { for } k=1,2, \ldots, n, \\
u_{k+1}-u_{k}=v_{k} l_{k} & \text { for } k=0,1,2, \ldots, n .
\end{aligned}
$$

It follows that, for $k=1,2, \ldots, n$,

$$
l_{k}^{-1}\left(u_{k+1}-u_{k}\right)-l_{k-1}^{-1}\left(u_{k}-u_{k-1}\right)=v_{k}-v_{k-1}=-\lambda u_{k} m_{k} .
$$

This shows that $\lambda$ is an eigenvalue of (2.3) with eigenvector $\left(u_{1}, u_{2}, \ldots, u_{n}\right)$. Retracing the steps one sees that eigenvalues of (2.3) are also eigenvalues of (2.5).

Remark 2. The eigenvalues $\lambda_{k}^{\prime}$ of the matrix eigenvalue problem $A^{\prime} z=\lambda M z$ are eigenvalues of (2.5) but with the boundary conditions $u(0)=v(b)=0$. So we can prescribe the spectra (2.2) for the boundary conditions $u(0)=u(b)=0$ and $u(0)=v(b)=0$ simultaneously.

\section{REFERENCES}

[1] F. V. Atkinson, "Discrete and Continuous Boundary Value Problems", Academic Press, New York, 1964. MR0176141 (31:416)

[2] P. Binding and H. Volkmer, "Prüfer angle asymptotics for Atkinson's semi-definite SturmLiouville eigenvalue problem", to appear in Math. Nachr.

[3] F. P. Gantmacher and M. G. Krein, "Oscillation Matrices and Kernels and Small Vibrations of Mechanical Systems", Amer. Math. Soc. Chelsea Publishing, Providence, Rhode Island, (2002). MR1908601 (2003f:34161)

[4] Q. Kong, H. Wu and A. Zettl, "Sturm-Liouville Problems with finite spectrum," J. Math. Anal. and Appl., (2001), 748-762. MR.1866077(2002g:34056)

[5] H. Volkmer, "Eigenvalues associated with Borel sets", to appear in Real Analysis Exchange.

[6] H. Volkmer, "Eigenvalue problems of Atkinson, Feller and Krein, and their mutual Relationship", Electron. J. Differential Equations 2005, No. 48, 15 pp. MR2135259 (2005k:34103)

[7] A. Zettl, "Sturm-Liouville Theory", Mathematical Surveys and Monographs, v. 121, Amer. Math. Soc. 2005. MR2170950

Department of Mathematical Sciences, University of Wisconsin, Milwaukee, WisconSIN 53201

E-mail address: volkmer@csd.uwm.edu

Department of Mathematics, Northern Illinois University, De Kalb, Illinois 60115

E-mail address: zettl@math.niu.edu 